\title{
Increased serum dehydroepiandrosterone sulfate in the first episode but not in subsequent episodes in male patients with schizophrenia
}

This article was published in the following Dove Press journal:

Neuropsychiatric Disease and Treatment

29 April 2014

Number of times this article has been viewed

\section{Murat Beyazyüz' \\ Yakup Albayrak ${ }^{2}$ \\ Elmas Beyazyüz ${ }^{3}$ \\ Cüneyt Ünsal ${ }^{2}$ \\ Erol Göka ${ }^{3}$}

'Department of Psychiatry, Gölbașı Hasvak State Hospital, Ankara, ${ }^{2}$ Department of Psychiatry, School of Medicine, Namık Kemal University, Tekirdağ, ${ }^{3}$ Department of Psychiatry, Ankara Numune Education and Research Hospital, Ankara, Turkey
Background: Many studies have investigated the relationship between blood levels of dehydroepiandrosterone (DHEA) and its sulfate ester (DHEA-S), cortisol, progesterone, and testosterone and the onset, prognosis, symptom severity, and treatment response of schizophrenia. In the present study, we assessed potential differences in blood levels of neurosteroids between drug-naïve first-episode patients with schizophrenia (FES), and drug-free patients with schizophrenia who were not in the first episode but were in a phase of acute exacerbation (DFP).

Materials and methods: The present study included 32 male FES, 28 male DFP, and 24 male healthy controls (HC). Groups were compared in terms of blood levels of adrenocorticotropic hormone (ACTH), cortisol, testosterone, progesterone, and DHEA-S.

Results: Blood levels of ACTH, cortisol, testosterone, and progesterone were similar among the groups. The mean value of serum DHEA-S was significantly different among the groups $(P<0.001)$. The value of serum DHEA-S was higher in the FES group than in the DFP and HC groups (both $P<0.001$ ). The mean values of serum DHEA-S in the HC and DFP groups were found to be similar $(P=0.33)$.

Conclusion: We suggest that higher values of DHEA-S in the FES group compared with both the DFP and $\mathrm{HC}$ groups indicate that this neurosteroid response is unique to first-episode schizophrenia patients. Further studies are needed to investigate the difference in blood levels of neurosteroids in different groups in terms of age of diagnosis.

Keywords: neurosteroid, effect, psychosis

\section{Introduction}

Neuroactive steroids, such as cortisol, dehydroepiandrosterone (DHEA) and its sulfate ester (DHEA-S), progesterone, and testosterone, are the steroid hormones that have several notable roles in the central nervous system. ${ }^{1,2}$ In the last 3 decades, several authors have posited a link between neuroactive steroids and the pathophysiology or therapeutics of schizophrenia. The relationship between blood levels of DHEA, DHEA$\mathrm{S}$, cortisol, progesterone, and testosterone and the onset, prognosis, symptom severity, and treatment response of schizophrenia has been investigated in a number of studies. ${ }^{3-5}$ The results of these studies have been inconsistent, but discussions about the impact of neuroactive steroids in the pathophysiology of schizophrenia are intriguing.

The sulfated form of DHEA is the most abundant neuroactive steroid in the blood. ${ }^{6,7}$ DHEA and DHEA-S regulate neuronal activity by means of slow mechanisms, such as gene expression, and by means of more rapid mechanisms, such as membrane-bound ligand-gated ion-channel interactions. ${ }^{1,8}$ DHEA and DHEA-S have been considered
Correspondence: Yakup Albayrak of Medicine, Namık Kemal University, Tunca Caddesi, Tekirdağ, 59100, Turkey Tel +905056355434 Fax +90282 2509950 Email dr.fuge@hotmail.com 
to have antistress and neuroprotective properties. ${ }^{2}$ Some studies have reported that the blood levels of these neuroactive steroids were lower in patients with schizophrenia than in healthy controls, but other studies have found elevated levels in patients with schizophrenia., ${ }^{4,5}$ These contradictory results make it difficult to form a hypothesis about the aforementioned relationships.

There are also inconsistent findings about the relationships between pathophysiology, prognosis, and symptom severity of schizophrenia and blood levels of progesterone, testosterone, and cortisol. ${ }^{10-12}$ Most of the studies in this subfield investigated these relationships by measuring blood levels of patients with schizophrenia, regardless of their treatment status, the number of past episodes, and other confounding factors. ${ }^{3,13-16}$ Moreover, patients with schizophrenia were frequently compared with healthy subjects. These studies did not measure alterations of blood levels of neuroactive steroids in different phases of the illness or compare blood levels of first-episode and later-episode patients. In the present study, we assessed potential differences in blood levels of DHEA-S, adrenocorticotropic hormone (ACTH), testosterone, progesterone, and cortisol between drug-naïve first-episode patients with schizophrenia (FES) and drug-free patients with schizophrenia who were not in the first episode but were in a phase of acute exacerbation (DFP).

\section{Materials and methods}

\section{Participants}

This study was conducted in the inpatient clinic at the Department of Psychiatry, Ankara Numune Research and Education Hospital. Sixty male patients who were diagnosed with schizophrenia according to the Diagnostic and Statistical Manual of Mental Disorders (DSM)-IV-TR criteria ${ }^{17}$ were included in the study. Among them, 32 patients were experiencing their first episode of schizophrenia and had never been exposed to any antipsychotic drug (FES group). The other 28 patients had been diagnosed with schizophrenia at least 2 years prior, and were in an acute exacerbation stage of schizophrenia due to treatment nonadherence (DFP group). All of these 28 patients had been drug-free for at least 4 weeks for oral antipsychotics and at least 6 weeks for long-acting injectable antipsychotics. The diagnoses were made by senior psychiatrists. Twenty-four male and age-matched healthy control subjects (HC) were selected to provide blood samples.

The study was approved by the local ethics committee at the Ankara Numune Research and Education Hospital. All subjects provided written informed consent for participation in the study after the procedure had been fully explained.
The exclusion criteria were 1) female sex, 2) the presence of any other psychiatric morbidity, such as alcohol or substance dependence, 3 ) the presence of any concurrent medical or endocrine disorder, and 4) the administration of other medications that could alter neurosteroid levels.

\section{Procedure}

All patients were clinically examined and individually interviewed. To obtain an objective history of the patients, accompanying close relatives were also interviewed. The patients were rated with the Scale for the Assessment of Negative Symptoms (SANS) ${ }^{18}$ and the Scale for the Assessment of Positive Symptoms (SAPS). ${ }^{19}$ Before initiating any pharmacological treatment, $10 \mathrm{~mL}$ of venous blood was collected at 8 am and divided into one tube with $2 \%$ heparin and another tube with ethylenediaminetetraacetic acid; this procedure was necessary to measure ACTH. Plasma levels of ACTH (normal range 7.2-63.3 pg/mL), cortisol (normal range 6.7-22.6 $\mu \mathrm{g} / \mathrm{dL}$ ), testosterone (normal range $8.9-42.5 \mathrm{pg} / \mathrm{mL}$ ), progesterone (normal range 0.14-2.06 ng/mL), and DHEA-S (normal range $85-690 \mu \mathrm{g} / \mathrm{dL}$ ) were measured by radioimmunoassay. Plasma levels of ACTH, cortisol, testosterone, progesterone, and DHEA-S were also collected from the consenting healthy subjects and measured using the same assay. To avoid interassay variability, the hormone levels in all groups were measured simultaneously.

\section{Psychopathological assessment instruments}

\section{Structured Clinical Interview for DSM-IV Axis I}

The Structured Clinical Interview for DSM-IV Axis I is a semistuctured interview for making major DSM-IV Axis I diagnoses. The instrument is designed to be administered by a clinician or trained mental health professional. It was developed by First et al, ${ }^{20}$ and the Turkish version was reported to be reliable by Corapcioglu et al. ${ }^{21}$

\section{Scale for the Assessment of Negative Symptoms}

The SANS assesses five symptom complexes to obtain clinical ratings of negative symptoms in patients with schizophrenia. These are affective blunting, alogia (impoverished thinking), avolition/apathy, anhedonia/asociality, and disturbance of attention. The final symptom complex seems to have less obvious relevance to negative symptoms than the other four complexes. Assessments are conducted on a 6-point scale $(0=$ not at all to $5=$ severe $)$. The instrument was developed by Andreasen. ${ }^{18}$ The Turkish version was reported to be reliable by Erkoç et al. ${ }^{22}$ 
Scale for the Assessment of Positive Symptoms

The SAPS was designed to assess positive symptoms, principally those that occur in schizophrenia. The instrument is intended to complement the SANS. The assessed positive symptoms include hallucinations, delusions, bizarre behavior, and positive formal thought disorder. The SAPS was developed by Andreasen..$^{19}$ The Turkish version was reported to be reliable by Erkoç et al. ${ }^{23}$

\section{Statistical methods}

The data were analyzed using the SPSS version 16.0 (SPSS Inc., Chicago, IL, USA). When possible, results were presented with $95 \%$ confidence intervals (CIs), and two-tailed $P$-values of less than 0.05 were considered to be statistically significant for all analyses. The variables were tested for homogeneity of variance using Levene's test, and for normality of distribution with the Kolmogorov-Smirnov test. Differences among age, serum levels of ACTH, cortisol, testosterone, progesterone, and DHEA-S were tested with a series of one-way analyses of variance. A Bonferroni test was applied in a post hoc analysis for multiple comparisons of the three groups. Student's $t$-test was used for comparing SANS and SAPS scores between the FES and DFP patients. Differences in smoking status were compared with $\chi^{2}$ tests. Pearson correlation was performed to analyze the correlation coefficients among age, SANS and SAPS scores, duration of treatment, serum levels of ACTH, cortisol, testosterone, progesterone, and DHEA-S. Linear regression analysis was used to identify associations between age and levels of serum neurosteroids.

\section{Results}

\section{Sociodemographic and clinical characteristics of the participants}

All participants were male. The mean ages of the FES, DFP, and HC patients were 25.24 $\pm 5.65,28.92 \pm 4.81$, and $26.67 \pm 5.19$ years, respectively. The mean age was younger in the FES group than in the DFP group $(F=3.58$, $P=0.033)$. Smoking status was similar among groups. The mean score of the SANS was higher in the DFP group than in the other groups $(t=-2.25, P=0.02)$. The difference in the mean scores of the SAPS between the FES and DFP groups was not statistically significant $(t=-1.62, P=0.10)$ (Table 1$)$.

\section{Serum ACTH, cortisol, testosterone, progesterone, and DHEA-S levels in the FES, DFS, and HC groups}

The mean values of serum ACTH, cortisol, progesterone, and testosterone levels in the FES, DFP, and HC groups were similar. The mean serum DHEA-S levels were significantly different among the groups ( $F=29.13, P<0.001)$. A post hoc Bonferroni test revealed that the value of serum DHEA-S was higher in the FES group $(330.68 \pm 78.87 \mu \mathrm{g} / \mathrm{dL})$ than in the DFP group $(132.87 \pm 44.97 \mu \mathrm{g} / \mathrm{dL}, P<0.001)$ and the $\mathrm{HC}$ group $(179.81 \pm 52.82 \mu \mathrm{g} / \mathrm{dL}, P<0.001)$. The mean values of serum DHEA-S were found to be similar between the DFP and $\mathrm{HC}$ groups $(P=0.33)$ (Table 2). Because the mean age was significantly different among the groups, regression was used to assess the association between age and plasma neurosteroids. There was only a significant association between level of serum cortisol and age in the HC group ( $P=0.02$; adjusted $R^{2}=0.240,0.355$, and 0.453 in the FES, DFP, and HC groups respectively).

\section{Correlations between age and SAPS and SANS scores and levels of plasma neurosteroids in the FES group}

There was a negative correlation between the serum cortisol level and mean age $(r=-0.523, P<0.001)$. There was a significant positive correlation between the mean SANS

Table I Clinical characteristics of participants

\begin{tabular}{|c|c|c|c|c|}
\hline & FES $(n=32)$ & DFP $(n=28)$ & $\mathrm{HC}(n=24)$ & Statistic \\
\hline \multirow[t]{4}{*}{ Age (years) } & $25.24 \pm 5.65$ & $28.92 \pm 4.81$ & $26.67 \pm 5.19$ & $F=3.58, P=0.033$ \\
\hline & & & & FES and DFP: $P=0.02$ \\
\hline & & & & FES and $\mathrm{HC}: P=0.96$ \\
\hline & & & & DFP and HC: $P=0.37$ \\
\hline \multicolumn{5}{|l|}{ Smoking } \\
\hline Yes & $29(87.5 \%)$ & 19 (67.9\%) & $15(62.5 \%)$ & $\chi^{2}=12.82, P=0.08$ \\
\hline No & $3(12.5 \%)$ & $9(32.1 \%)$ & 9 (37.5\%) & \\
\hline SANS & $30.96 \pm 7.05$ & $35.10 \pm 7.14$ & & $t=-2.25, P=0.02$ \\
\hline SAPS & $31.43 \pm 5.18$ & $33.78 \pm 6.01$ & & $t=-1.62, P=0.10$ \\
\hline Duration of treatment (years) & - & $5.78 \pm 3.12$ & - & - \\
\hline
\end{tabular}

Abbreviations: DFP, drug-free patients; FES, first-episode schizophrenia; HC, healthy controls; SANS, Scale for the Assessment of Negative Symptoms; SAPS, Scale for the Assessment of Positive Symptoms. 
Table 2 Comparisons of serum ACTH, cortisol, testosterone, progesterone, and DHEA-S levels between groups

\begin{tabular}{|c|c|c|c|c|}
\hline & FES $(n=32)$ & DFP $(n=28)$ & HC $(n=24)$ & Statistic \\
\hline Cortisol ( $\mu \mathrm{g} / \mathrm{dL})$ & $9.73 \pm 3.59$ & $\mid 0.08 \pm 3.31$ & $10.34 \pm 3.65$ & $F=0.87, P=0.91$ \\
\hline Progesterone $(\mathrm{ng} / \mathrm{mL})$ & $|.7| \pm 0.4 \mid$ & $1.62 \pm 0.33$ & $1.10 \pm 0.66$ & $F=0.78, P=0.46$ \\
\hline \multirow[t]{4}{*}{ DHEA-S $(\mu \mathrm{g} / \mathrm{dL})$} & $330.68 \pm 78.87$ & $132.87 \pm 44.97$ & $|79.8| \pm 52.82$ & $F=29.13, P<0.001$ \\
\hline & & & & FES and DFP: $P<0.00 I$ \\
\hline & & & & FES and $\mathrm{HC}: P<0.00 \mathrm{I}$ \\
\hline & & & & DFP and HC: $P=0.33$ \\
\hline ACTH $(p g / m L)$ & $15.01 \pm 8.49$ & $17.97 \pm 7.67$ & $14.65 \pm 9.08$ & $F=1.29, P=0.28$ \\
\hline Testosterone $(\mathrm{pg} / \mathrm{mL})$ & $14.57 \pm 6.49$ & $11.15 \pm 6.93$ & $9.74 \pm 4.71$ & $F=2.14, P=0.13$ \\
\hline
\end{tabular}

Abbreviations: ACTH, adrenocorticotropic hormone; DFP, drug-free patients; DHEA-S, dehydroepiandrosterone sulfate; FES, first-episode schizophrenia; HC, healthy controls.

score and the serum ACTH level $(r=0.352, P<0.05)$, and a negative correlation between the mean SANS score and the serum DHEA-S level $(r=-0.546, P<0.001)$. The mean SAPS score was negatively correlated with the mean values of serum testosterone $(r=-0.353, P<0.001)$ and cortisol $(r=-0.620, P<0.001)$ (Table 3).

\section{Correlations between age and SAPS and SANS scores, duration of treatment, and levels of plasma neurosteroids in the DFP group}

There was a negative correlation between the serum ACTH level and mean age $(r=-0.426, P<0.05)$ and a positive correlation between serum testosterone and mean age $(r=0.561$, $P<0.001)$. There was a significant positive correlation between the mean SANS score and serum ACTH and cortisol levels (ACTH: $r=0.490, P<0.001$; cortisol: $r=0.428, P<0.05$ ). The mean SAPS score was negatively correlated with the values of serum cortisol $(r=-0.415, P<0.001)$ and DHEA-S $(r=-0.465$, $P<0.001)$. The duration of treatment was negatively correlated with the mean values of DHEA-S $(r=-0.390, P<0.05)$ and $\operatorname{ACTH}(r=-0.560, P<0.001)$, and positively correlated with the mean serum testosterone level $(r=0.673, P<0.001)$ (Table 4).

\section{Discussion}

Schizophrenia is a chronic and debilitating disorder characterized by various symptoms. Elevated emotional distress, cognitive decline, and deterioration in social functioning are core symptoms in schizophrenia. ${ }^{24}$ Regardless of the intensity of symptoms at the onset, diverse periods with variation in symptoms and symptom severity may occur during the course of the illness. As with other chronic medical conditions, patients who have the same diagnosis seldom experience the same symptoms over similar time periods. A patient with schizophrenia may experience a residual or remission period; the same patient may have been under treatment or drug-free for months. Moreover, the patient may be in an acute exacerbation period that involves acute and severe symptoms. This exacerbation may occur in spite of an effective treatment or as a result of giving up a treatment. Therefore, the validity of data that are obtained by comparing patients in different phases of a disease is questionable.

In this study, the most salient finding was higher blood levels of DHEA-S in the FES group compared to the DFP and HC groups. Notably, patients in the DFP group were not in remission or a residual period, but were in a state of acute exacerbation. Previous studies on the blood levels of DHEA or DHEA-S in patients with schizophrenia or other psychotic spectrum disorders have shown inconsistent results. Serum levels of DHEA and DHEA-S have been reported to be lower, ${ }^{25,26}$ similar, $^{3,27}$ and elevated ${ }^{13-15}$ in patients with schizophrenia. Several studies found increased blood levels of DHEA-S in FES compared to healthy controls, but the authors argued that this could not be seen in chronic patients;

Table 3 Correlation coefficients between scores of SAPS, SANS, and DT, and levels of serum ACTH, cortisol, testosterone, progesterone, and DHEA-S in the FES group

\begin{tabular}{llllll}
\hline & Cortisol & Progesterone & DHEA-S & ACTH & Testosterone \\
\hline Age & $-0.523 * *$ & 0.103 & 0.004 & -0.013 & -0.002 \\
SANS & -0.104 & -0.166 & $-0.546 * *$ & $0.352^{*}$ & 0.189 \\
SAPS & $-0.353^{* *}$ & 0.048 & -0.295 & 0.135 & $-0.620 * *$ \\
\hline
\end{tabular}

Notes: ${ }^{* * P}<0.001 ; * P<0.05$.

Abbreviations: ACTH, adrenocorticotropic hormone; DFP, drug-free patients; DHEA-S, dehydroepiandrosterone sulfate; DT, duration of treatment; FES, first-episode schizophrenia; HC, healthy controls; SANS, Scale for the Assessment of Negative Symptoms; SAPS, Scale for the Assessment of Positive Symptoms. 
Table 4 Correlation coefficients between scores of SAPS, SANS, and DT, and levels of serum ACTH, cortisol, testosterone, progesterone, and DHEA-S in the DFP group

\begin{tabular}{|c|c|c|c|c|c|}
\hline & Cortisol & Progesterone & DHEA-S & АСТH & Testosterone \\
\hline Age & 0.072 & -0.039 & -0.145 & $-0.426 *$ & $0.561 * *$ \\
\hline SANS & $0.428^{*}$ & -0.310 & -0.081 & $0.490 * *$ & 0.188 \\
\hline SAPS & $-0.415^{* *}$ & -0.017 & $-0.465 * *$ & 0.122 & -0.036 \\
\hline DT & 0.052 & -0.011 & $-0.390 *$ & $-0.560 * *$ & $0.673 * *$ \\
\hline
\end{tabular}

Notes: $* * P<0.001 ; * P<0.05$.

Abbreviations: ACTH, adrenocorticotropic hormone; DFP, drug-free patients; DHEA-S, dehydroepiandrosterone sulfate; DT, duration of treatment; FES, first-episode schizophrenia; HC, healthy controls; SANS, Scale for the Assessment of Negative Symptoms; SAPS, Scale for the Assessment of Positive Symptoms.

to our knowledge, no study has compared the blood levels of neurosteroids in male FES with those in male DFP. Therefore, previous research provides little evidence for assertions that higher levels of DHEA-S reflect a neuroprotective response to psychosis that becomes blunted as the illness becomes more chronic. However, our results provide evidence for this conclusion.

The findings of this study are consistent with previous interpretations (see especially Strous et al) ${ }^{14,15}$ suggesting that FES exhibit a neurosteroid response to psychosis. Higher values of DHEA-S levels in the FES group compared to both the DFP and HC groups indicate that this neurosteroid response is peculiar to FES patients. Neuroactive steroids, especially DHEA and DHEA-S, have long been known to have neuroprotective effects. ${ }^{28-31}$ If elevated levels of these substances in the blood serve as neuroendocrinological adaptive or protective mechanisms, they would provide a one-time service for patients with schizophrenia. If this is the case, then treatment decisions for patients with schizophrenia should differ for single-episode versus chronic patients. An intrinsic protective mechanism may not occur after the first episode. There is no evidence that the mechanism is related to drug use, as this study shows that the blood levels of DHEA-S were lower in the DFP group than in the FES group; levels of neuroactive steroids may be diminished in subsequent episodes of the illness. In the present study, the decision to measure DHEA-S without DHEA reflects the fact that DHEA-S is the most abundant neuroactive steroid in circulation and a metabolite of DHEA. DHEA is a short-life molecule, and is metabolized rapidly to DHEA-S. ${ }^{32}$ Therefore, the levels of DHEA-S reflect the levels of DHEA, and increased DHEA-S levels indicate that DHEA levels recently increased.

Distress is known to cause increases in blood levels of neurosteroids. ${ }^{33-35}$ In other psychiatric conditions that are accompanied by serious distress, blood levels of DHEA and DHEA-S were found to be elevated. ${ }^{36,37}$ Therefore, the question is which neurosteroid response is specific to which psychotic episode. Stress nonspecifically increases the blood levels of cortisol. In our study, there were no significant differences in serum ACTH or cortisol levels among the groups. Several neuroendocrinological studies emphasize that an uncertain dysfunction of the hypothalamic-pituitary-adrenal axis plays a role in the pathophysiology of schizophrenia, ${ }^{38,39}$ but there is insufficient evidence of this role in patients with schizophrenia. Given the variation on the schizophrenia spectrum, the study discrepancies in terms of blood levels of $\mathrm{ACTH}$ and cortisol are far from being severe. However, in our study, no significant differences in blood cortisol and ACTH levels were found among the groups; the similarities among the three groups show that the neurosteroid response is not a nonspecific response to stress. Nevertheless, in this study, the FES and DFP groups were similar in terms of symptom severity. Therefore, the results of this study are consistent with early suggestions about a diminished neurosteroid response in the later periods of illness.

In the present study, there were no significant differences in blood levels of testosterone and progesterone among the groups. Because various results have been found in previous studies, this finding should not be overinterpreted. In some studies, sample groups were taking medication, and the blood levels of some hormones may have been affected by the antipsychotics. In other studies, only patients with negative symptoms were considered. Oades and Schepker ${ }^{13}$ investigated serum steroid hormones in young patients with schizophrenia, and found no differences in blood levels of testosterone and progesterone in young male patients with schizophrenia compared to healthy controls and patients with obsessive compulsive disorder. However, their patients were not drug-naïve or drug-free, and many antipsychotic drugs are known to interfere with these measurements. Likewise, in their study, Shirayama et $\mathrm{al}^{37}$ found that the progesterone levels of patients with schizophrenia were similar with those of healthy controls, but they found that the testosterone levels were lower in the group of patients with moderate negative symptoms. Similarly, Goyal et al ${ }^{26}$ found no difference in blood levels of testosterone between 
patients with schizophrenia and healthy controls. Most of these studies included patient groups that were under medical treatment. In our study, neither patient groups was receiving any drug treatment; this criterion was selected so that the groups would be as homogeneous as possible. For the same reason, female patients were excluded from this study, as blood levels of some hormones, including progesterone, are known to vary during the menstrual period. Blood samples must be taken from female patients on the same days of the menstrual cycle to achieve valid results. This requirement is difficult and demanding for clinicians. Moreover, males are known to demonstrate higher DHEA-S levels. ${ }^{40}$ Late-onset schizophrenia and a better prognosis among females are patterns in need of explanation, so there would have been many confounding factors if females had been included in the study.

Researching peripheral blood biomarkers for schizophrenia enhances the potential to discover diagnostic and prognostic indicators of utility and to understand the pathophysiology of schizophrenia. ${ }^{41}$ In addition to neuroendocrinological and neuroimaging studies that investigated potential biomarkers for schizophrenia, there are several studies that have explored immunological factors and the association between schizophrenia and antipsychotic therapy. Himmerich et al reported that there were significant impacts of antipsychotics on interleukin levels in vivo. ${ }^{42}$ Another study by Borovcanin et al, in which the grouping of participants was similar to our study, reported that type II cytokine levels were elevated, and there were low interleukin 17 levels in FES patients and patients with schizophrenia who were in relapse. ${ }^{43}$ In a review, Schmidt et al noted the roles of eicosanoids and related enzymes in the etiology and treatment of schizophrenia. ${ }^{44}$ In addition to highlighting the presence of neuroprotective and stress-response roles of elevated DHEA-S levels in FES patients, elevated DHEA-S may be considered to be a biomarker for schizophrenia. However, further studies are needed to identify the biomarker role of DHEA-S in schizophrenia.

There are several limitations of the present study. The major limitation is its design. Comparing neurosteroids in the same first-episode and later-episode schizophrenia patients may be the best way to achieve reliable results. However, comparing biomarkers in patients with schizophrenia in their first episode and in subsequent episodes may be impossible to achieve while the patients remain drug-free. We could not investigate blood levels of antipsychotics, so our antipsychotic treatment data were obtained from patients and their first-degree relatives. Because of our study design, only male patients were included in the study, which may be considered to be a limitation. Another limitation is that patients who were suffering from their first episode of schizophrenia were younger, which is to be expected. Finally, patients with obesity were not included in the study, and we have no data that would determine whether body mass index has an association with serum neurosteroid levels.

In conclusion, our study provides valid evidence in support of previous hypotheses in this field of research. Further prospective studies should investigate the differences in blood levels of neurosteroids in patients with schizophrenia.

\section{Disclosure}

The authors report no conflicts of interest in this work.

\section{References}

1. Paul SM, Purdy RH. Neuroactive Steroids. FASEB J. 1992;6:2311-2322.

2. Maninger N, Wolkowitz OM, Reus VI, Epel ES, Mellon SH. Neurobiological and neuropsychiatric effects of dehydroepiandrosterone (DHEA) and DHEA sulfate (DHEAS). Front Neuroendocrinol. 2009;30:65-91.

3. Ritsner M, Maayan R, Gibel A, Strous RD, Modai I, Weizman A. Elevation of the cortisol/dehydroepiandrosterone ratio in schizophrenia patients. Eur Neuropsychopharmacol. 2004;14:267-273.

4. Lerer B, Ran A, Blacker M, et al. Neuroendocrine responses in chronic schizophrenia. Evidence for serotonergic dysfunction. Schizophr Res. 1988;1:405-410.

5. Meltzer HY, Lee MA, Jayathilake K. The blunted plasma cortisol response to apomorphine and its relationship to treatment response in patients with schizophrenia. Neuropsychopharmacology. 2001;24:278-290.

6. Baulieu EE, Robel P. Dehydroepiandrosterone and dehydroepiandrosterone sulfate as neuroactive steroids. $J$ Endocrinol. 1996;150: 221-239.

7. Wolf OT, Neumann O, Hellhammer DH, et al. Effects of a two-week physiological dehydroepiandrosterone substitution on cognitive performance and well-being in healthy elderly women and men. J Clin Endocrinol Metab. 1997;82:2363-2367.

8. Rupprecht R, di Michelle F, Hermann B, et al. Neuroactive steroids: molecular mechanisms of action and implications for neuropsychopharmacology. Brain Res Brain Res Rev. 2001;37:59-67.

9. Ritsner MS. Pregnenolone, dehydroepiandrosterone, and schizophrenia: alterations and clinical trials. CNS Neurosci Ther. 2010;16:32-44.

10. Alexander GM, Peterson BS. Sex steroids and human behavior: implications for developmental psychopathology. CNS Spectr. 2001;6:75-88.

11. Walder DJ, Walker EF, Lewine RJ. Cognitive functioning, cortisol release, and symptom severity in patients with schizophrenia. Biol Psychiatry. 2000;48:1121-1132.

12. Brophy MH, Rush JA, Crowley G. Cortisol, estradiol, and androgens in acutely ill paranoid schizophrenics. Biol Psychiatry. 1983;18:583-590.

13. Oades RD, Schepker R. Serum gonadal steroid hormones in young schizophrenic patients. Psychoneuroimmunology. 1994;19: 373-385.

14. Strous RD, Maayan R, Lapidus R, et al. Increased circulatory dehydroepiandrosterone and dehydroepiandrosterone-sulphate in first-episode schizophrenia: relationship to gender, aggression and symptomatology. Schizophr Res. 2004;71:427-434. 
15. Strous RD, Maayan R, Kaminsky M, Blumensohn R, Weizman A, Spivak B. DHEA and DHEA-S levels in hospitalized adolescents with first-episode schizophrenia and conduct disorder: a comparison study. Eur Neuropsychopharmacol. 2009;19:499-503.

16. Yıldırım O, Dogan O, Semiz M, Kilicli F. Serum cortisol and dehydroepiandrosterone-sulfate levels in schizophrenic patients and their first-degree relatives. Psychiatry Clin Neurosci. 2011;65:584-591.

17. American Psychiatric Association. Diagnostic and Statistical Manual of Mental Disorders. 4th ed. Washington: American Psychiatric Association; 2000.

18. Andreasen NC. Scale for the Assessment of Negative Symptoms (SANS). Iowa City: College of Medicine, University of Iowa; 1984.

19. Andreasen NC. Scale for the Assessment of Positive Symptoms (SAPS). Iowa City: College of Medicine, University of Iowa; 1984.

20. First MB, Spitzer RL, Gibbon M, Williams JB. Structured Clinical Interview for DSM-IV Axis I Disorders (SCID-I): Clinician Version. Washington: American Psychiatric Press; 1996.

21. Corapcioglu A, Aydemir O, Yildiz M. [Structured clinical interview for DSM-IV (SCID-IV): Turkish version]. Ankara: Hekimler Yayin Birligi; 1999. Turkish.

22. Erkoç S, Arkonaç O, Ataklı C, Ozmen E. Negatif semptomları değerlendirme ölçeğinin güvenirliği ve geçerliliği. [The reliability and validity of scale for the assessment of negative symptoms.] Düşünen Adam. 1991;4:16-19.

23. Erkoç S, Arkonaç O, Ataklı C, Ozmen E. Pozitif semptomları değerlendirme ölçeğinin güvenirlirliği ve geçerliliği. [The reliability and validity of scale for the assessment of positive symptoms.] Düşünen Adam. 1991;4:20-24.

24. Bilder RM. Neurocognitive impairment in schizophrenia and how it affects treatment options. Can J Psychiatry. 1997;42:255-264.

25. Tourney G, Erb JL. Temporal variations in androgens and stress hormones in control and schizophrenic subjects. Biol Psychiatry. $1979 ; 14: 395-404$

26. Goyal RO, Sagar R, Ammini AC, Khurana ML, Alias AG. Negative correlation between negative symptoms of schizophrenia and testosterone levels. Ann N Y Acad Sci. 2004;1032:291-294.

27. Yilmaz N, Herken H, Cicek HK, Celik A, Yurekli M, Akyol O. Increased levels of nitric oxide, cortisol and adrenomedullin in patients with chronic schizophrenia. Med Princ Pract. 2007;16:137-141.

28. Maayan R, Yagorowski Y, Grupper D, et al. Basal plasma dehydroepiandrosterone sulfate level: a possible predictor for response to electroconvulsive therapy in depressed psychotic inpatients. Biol Psychiatry. 2000;48:693-701.

29. Kaasik A, Safiulina D, Kalda A, Zharkovsky A. Dehydroepiandrosterone sulphate prevents oxygen-glucose deprivation-induced injury in cerebellar granule cell culture. Neuroscience. 2001;102:427-432.
30. Kimonides VG, Khatibi NH, Svendsen CN, Sofroniew MV, Herbert J. Dehydroepiandrosterone (DHEA) and DHEA-sulfate (DHEAS) protect hippocampal neurons against excitatory amino acid-induced neurotoxicity. Proc Natl Acad Sci U S A. 1998;95:1852-1857.

31. Zhang L, Li B, Ma W, et al. Dehydroepiandrosterone (DHEA) and its sulfated derivative (DHEAS) regulate apoptosis during neurogenesis by triggering the Akt signalling pathway in opposing ways. Brain Res Mol Brain Res. 2002;98:58-66.

32. Rosenfeld RS, Rosenberg BJ, Fukushima DK, Hellman L. 24-Hour secretory pattern of dehydroisoandrosterone and dehydroisoandrosterone sulfate. Clin Endocrinol Metab. 1975;40:850-855.

33. Corpéchot C, Robel P, Axelson M, Sjövall J, Baulieu EE. Characterization and measurement of dehydroepiandrosterone sulfate in rat brain. Proc Natl Acad Sci U S A. 1981;78:4704-4707.

34. Oberbeck R, Benschop RJ, Jacobs R, et al. Endocrine mechanisms of stress-induced DHEA secretion. J Endocrinol Invest. 1998;21:148-153.

35. Söndergaard HP, Hansson LO, Theorell T. Elevated blood levels of dehydroepiandrosterone sulphate vary with symptom load in posttraumatic stress disorder: findings from a longitudinal study of refugees in Sweden. Psychother Psychosom. 2002;71:298-303.

36. Spivak B, Maayan R, Kotler M, et al. Elevated circulatory levels of GABA(A)-antagonistic neurosteroids in patients with combat-related post-traumatic stress disorder. Psychol Med. 2000;30:1227-1231.

37. Shirayama Y, Hashimoto K, Suzuki Y, Higuchi T. Correlation of plasma neurosteroid levels to the severity of negative symptoms in male patients with schizophrenia. Schizophr Res. 2002;58:69-74.

38. Marx CE, Lieberman JA. Psychoneuroendocrinology of schizophrenia. Psychiatr Clin North Am. 1988;21:413-434.

39. Walker EF, Diforio D. Schizophrenia: a neural diathesis-stress model. Psychol Rev. 1997;104:667-685.

40. Orentreich N, Brind JL, Vogelman JH, Andres R, Baldwin H. Long term longitudinal measurements of plasma dehydroepiandrosterone sulfate in normal men. J Clin Endocrinol Metab. 1992;75:1002-1004.

41. Chana G, Bousman CA, Money TT, et al. Biomarker investigations related to pathophysiological pathways in schizophrenia and psychosis. Front Cell Neurosci. 2013;7:95.

42. Himmerich H, Schönherr J, Fulda S, Sheldrick AJ, Bauer K, Sack U. Impact of antipsychotics on cytokine production in-vitro. J Psychiatr Res. 2011;45:1358-1365.

43. Borovcanin M, Jovanovic I, Radosavljevic G, et al. Elevated serum level of type-2 cytokine and low IL-17 in first episode psychosis and schizophrenia in relapse. J Psychiatr Res. 2012;46:1421-1426.

44. Schmidt L, Ceglarek U, Kortz L, et al. Mechanisms of involvement of eicosanoids and their precursors in the pathophysiology and treatment of schizophrenia. Med Chem. 2013;9:763-773.
Neuropsychiatric Disease and Treatment

\section{Publish your work in this journal}

Neuropsychiatric Disease and Treatment is an international, peerreviewed journal of clinical therapeutics and pharmacology focusing on concise rapid reporting of clinical or pre-clinical studies on a range of neuropsychiatric and neurological disorders. This journa is indexed on PubMed Central, the 'PsycINFO' database and CAS

\section{Dovepress}

The manuscript management system is completely online and includes a very quick and fair peer-review system, which is all easy to use. Visit http://www.dovepress.com/testimonials.php to read real quotes from published authors. 